\title{
New abundance measurements in UKS 1927-177, a very metal-poor galaxy in the Local Group ${ }^{\star}$
}

\author{
I. Saviane ${ }^{1}$, L. Rizzi2 ${ }^{2,3}$, E. V. Held ${ }^{2}$, F. Bresolin ${ }^{4}$, and Y. Momany ${ }^{3}$ \\ ${ }^{1}$ European Southern Observatory, Casilla 19001, Santiago 19, Chile \\ 2 Osservatorio Astronomico di Padova, vicolo dell'Osservatorio 5, 35122 Padova, Italy \\ e-mail: (rizzi, held)@pd.astro.it \\ 3 Dipartimento di Astronomia, Università di Padova, vicolo dell'Osservatorio 2, 35122 Padova, Italy \\ e-mail: momany@pd.astro.it \\ ${ }^{4}$ Institute for Astronomy, University of Hawaii, 2680 Woodlawn Drive, Honolulu, HI 96822 \\ e-mail: bresolin@ifa.hawaii.edu
}

Received 26 February 2002 / Accepted 15 May 2002

\begin{abstract}
We present new results from optical spectroscopy of the brightest HII region in the dwarf irregular galaxy UKS 1927-177 in Sagittarius (SagDIG). From high signal-to-noise spectra, reddening-corrected line flux ratios have been measured with typical uncertainties of a few percent, from which the oxygen abundance is rediscussed, and new abundance estimates are derived for $\mathrm{N}$ and $\mathrm{Ne}$. The $\mathrm{O}$ abundance in SagDIG, estimated with the empirical abundance indicator $R_{23}$ and other methods, is in the range $12+\log (\mathrm{O} / \mathrm{H})=7.26$ to 7.50 . The fact that SagDIG is $\sim 10$ times closer than IZw 18 makes it an ideal target to test the hypothesis of the existence of young galaxies in the present-day universe. Indeed, stellar photometry suggests that this galaxy may harbor a stellar population older than a few Gyr, and possibly an old stellar component as well. The case of SagDIG therefore supports the view that very low chemical abundances can be maintained throughout the life of a dwarf stellar system, even in the presence of multiple star formation episodes.
\end{abstract}

Key words. galaxies: individual: SagDIG - galaxies: abundances - galaxies: dwarf - galaxies: ISM - galaxies: irregular Local Group

\section{Introduction}

The quest for very metal-poor galaxies started soon after the Searle \& Sargent (1972) discovery that the interstellar medium (ISM) of I Zw 18, a blue compact dwarf galaxy, has an oxygen abundance that is $\sim 1 / 50$ of the solar one. As Searle \& Sargent (1972) pointed out, this is compatible with two scenarios: either the galaxy is now experiencing its first star-formation (SF) episode, or, if it contains $\sim 10 \mathrm{Gyr}$ old stars, then its SF proceeds in strong bursts separated by quiescent phases. The first hypothesis (that of a primordial galaxy) is relevant in the context of cosmological models that assume hierarchical formation of structures in a cold dark matter dominated Universe. In that scenario, dwarf dark matter halos $\left(\sim 10^{8} M_{\odot}\right)$ would be the first objects to condense out of the Hubble flow, so it is important to understand whether they already contained stars before merging into larger galaxies. As a first step, we can start by answering a simple question: is there any galaxy with a very metal-poor young population and, at the same time, a $>10 \mathrm{Gyr}$ old generation of stars? Such a finding would lead to the

Send offprint requests to: I. Saviane,

e-mail: isaviane@eso.org

* Based on data collected at the European Southern Observatory, La Silla, Chile, prop. No. 63.N-0726B. conclusion that very low metal abundances do not necessarily imply a very young age.

Although a red population was eventually revealed in IZw 18 by Aloisi et al. (1999), their data only allow for a lookback time of $\sim 1 \mathrm{Gyr}$, due to the large distance of the galaxy. Izotov et al. (2001) argue that $100 \mathrm{Myr}$ is a more realistic limit. As a consequence, active searches are carried out to find closer and comparably metal-poor galaxies, in order to have better chances to detect a cosmologically old population. In particular, low luminosity dwarf irregulars are promising candidates. It is still debated whether a definite luminosity-metallicity relation exists for dIrrs (Hidalgo-Gamez \& Olofsson 1998; Hunter \& Hoffman 1999), but it is nevertheless reasonable to expect that chemical abundances will be lower in low-luminosity galaxies.

Indeed, some new metal-poor, low-luminosity objects turned up in the past as a result of similar efforts. The situation has been conveniently summarized by Kniazev et al. (2000), who list two galaxies (besides IZw 18) at $12+\log (\mathrm{O} / \mathrm{H}) \leq$ 7.35. A few months later van Zee (2000) added UGCA 292 to the list of metal-poor galaxies, quoting $12+\log (\mathrm{O} / \mathrm{H})=$ $7.30 \pm 0.05$, and pointed out that Leo A should be added to the list as well. 
Stimulated by all these promising results, we turned our attention to UKS 1927-177 (SagDIG), since (a) its low luminosity is in the range of other metal-poor galaxies, (b) there was some indication that the oxygen abundance obtained by Skillman et al. (1989b, hereafter STM89), $12+\log (\mathrm{O} / \mathrm{H})=$ 7.41 , could be revised downwards (see below), and (c) the other metal-poor galaxies (with the exception of Leo A) are more distant than $3 \mathrm{Mpc}$. SagDIG is more than two magnitudes closer in distance modulus (thus its HB is within reach of HST observations in reasonable observing time).

The galaxy was discovered in 1977 by Cesarsky et al. (1977), and a shallow CMD was presented in Cook (1987). A CCD survey of its HII regions was carried out by Strobel et al. (1991; SHK91), using the $2.1 \mathrm{~m}$ telescope at Kitt Peak. Three objects were detected in a field of view of $\sim 2.5 \times 2.5$, with $\mathrm{H} \alpha$ fluxes of $28.1 \times 10^{-15}, 20.0 \times 10^{-15}$, and $4.09 \times$ $10^{-15} \mathrm{erg} \mathrm{cm}^{-2} \mathrm{~s}^{-1}$. Optical spectrophotometry of the brightest object (object \#3 in Table 8 of SHK91) was obtained by STM89, and an oxygen abundance $\sim 1 / 30$ of the solar value ${ }^{1}$ could be determined, i.e. $12+\log (\mathrm{O} / \mathrm{H}) \simeq 7.41$. Radio maps of the galaxy exist as well. Young \& Lo (1997) presented deep VLA observations of the Hr in SagDIG. An amount of $1.3 \times 10^{7}$ solar masses of $\mathrm{H}+\mathrm{He}$ were detected, forming a circular ring which extends $\sim 3$ times farther than the optical extent of the star-forming regions.

The compactness of the galaxy, its low Galactic latitude, and the high foreground contamination had discouraged further broadband photometry until recently. In the last two years, imaging of this galaxy was presented by Karachentsev et al. (1999) and Lee \& Kim (2000), who established the distance modulus at $(m-M)_{0}=25.13 \pm 0.25$, and the RGB stellar metallicity at extremely low values $([\mathrm{Fe} / \mathrm{H}] \lesssim-2.45)$. From surface photometry, Karachentsev et al. (1999) found an integrated magnitude of $M_{V}=-11.74$, and with a synthetic CMD analysis, they concluded that the galaxy is currently experiencing a burst of star formation, the star formation rate (SFR) being 10 times larger now than the life average.

A new imaging study of SagDIG is presented in a companion paper (Momany et al. 2001). The most important results of our photometry is represented by an upward revision of the stellar metallicity of the galaxy and our analysis of the spatial distribution of different stellar populations as a function of age. Assuming a low reddening for old RGB stars, we derived $[\mathrm{Fe} / \mathrm{H}]=-2.08 \pm 0.20$ for the red giant branch stars.

In this paper we present our new determination of the oxygen, nitrogen, and neon abundances in the interstellar medium of UKS 1927-177, and discuss it in the context of the existence of primordial galaxies.

\section{Observations and data reduction}

Spectroscopic observations of SagDIG were carried out on 1999 August 10, at the ESO observatory in La Silla, Chile. The imaging spectrograph EFOsc2 attached to the

\footnotetext{
${ }^{1}$ In this paper we adopt the classical value $12+\log (\mathrm{O} / \mathrm{H})=8.87$ from Anders \& Grevesse (1989). However, the solar oxygen abundance is currently debated, and a lower value might be appropriate (Holweger 2001; Allende Prieto et al. 2001).
}

ESO 3.6m telescope was used to secure low-resolution spectroscopy of SagDIG. The camera was equipped with a Loral/Lesser thinned, AR coated, UV flooded, $2048^{2}$ pixel CCD (ESO CCD \#40). The $15 \mu$ pixels cover 0 ' 157 on the sky, with a resulting field of view of 5'.4×5'4. Two grisms were employed to cover the wavelength ranges $3270 \div 5240 \AA$ (grism $\# 7$ ) and $4700 \div 6770 \AA$ (grism \#9), respectively. The CCD was used in $2 \times 2$ binning mode to increase the $S / N$ ratio, the resulting dispersion being $\sim 2 \AA /$ pixel for both grisms.

We observed the same HII region studied by STM89, whose coordinates are $\alpha=19: 30: 02.5, \delta=-17: 41: 26.1$ (J2000.0). The night was stable and photometric, with a fairly constant seeing of $\sim 1^{\prime \prime}(F W H M)$ at $\mathrm{H} \alpha$. Spectroscopic data comprise exposures of $6 \times 1800 \mathrm{~s}$ with grism \#7 and $4 \times 1800 \mathrm{~s}$ using grism \#9. In both cases, a slit width of 1".5 was used. Spectrophotometric standards were observed during the night, selected from the catalogs of Oke (1990) and Hamuy et al. $(1992,1994)$.

Data reduction was performed using standard techniques in $\mathrm{IRAF}^{2}$. The raw two-dimensional images were bias subtracted and trimmed. Pixel-to-pixel variations were accounted for through division by a quartz lamp spectrum, after fitting a low-order polynomial along the dispersion. The splitting of the total exposure time in $1800 \mathrm{~s}$ exposures allowed us to remove the contamination from cosmic rays. Wavelength calibration was obtained from He-Ar exposures taken immediately before and after the object observations. Absolute flux calibration was achieved using standard stars, obtaining a final accuracy better than 5\%. One-dimensional spectra were extracted from the two-dimensional images by summing up all the pixels enclosed in the large profiles of $\mathrm{H} \alpha$ and $\mathrm{H} \beta$; the background and sky lines were subtracted by averaging a small region on either side of the extraction window.

Figure 1 shows the combined spectra used for measurements. The final resolution, measured either on sky lines or comparison lamp lines, is $8.3 \AA$ for grism \#7 and $9 \AA$ for grism \#9. Table 1 presents the final results. From left to right, the columns report the line wavelength and identification, the $\mathrm{H} \beta$-normalized fluxes for grisms \#7 and \#9, and the reddening corrected fluxes (see below). Three emission lines ([OIII] $\lambda \lambda 4959,5007$ and $\mathrm{H} \beta$ ) are in common between the two grisms, showing no significant flux offset. In the worst case, that of the [OIII] $\lambda 5007$ line, the agreement is better than $5 \%$. A deblending procedure was applied to the case of the [NII] $\lambda 6584$ line to disentangle the contribution of the strong $\mathrm{H} \alpha$. The error budget was estimated from the rms of line ratios measured on the different individual exposures. A maximum error of $15 \%$ on the flux ratio was attached to our estimate when the line is not detected in the single-exposure spectra.

The measured line ratios were corrected for the effects of reddening, measured by comparing the observed and the expected hydrogen line ratios. The theoretical $\mathrm{H}$ line ratios of Hummer \& Storey (1987) were used, assuming an electron

\footnotetext{
${ }^{2}$ IRAF is distributed by the National Optical Astronomy Observatories, which are operated by the Association of Universities for Research in Astronomy, Inc., under cooperative agreement with the National Science Foundation.
} 

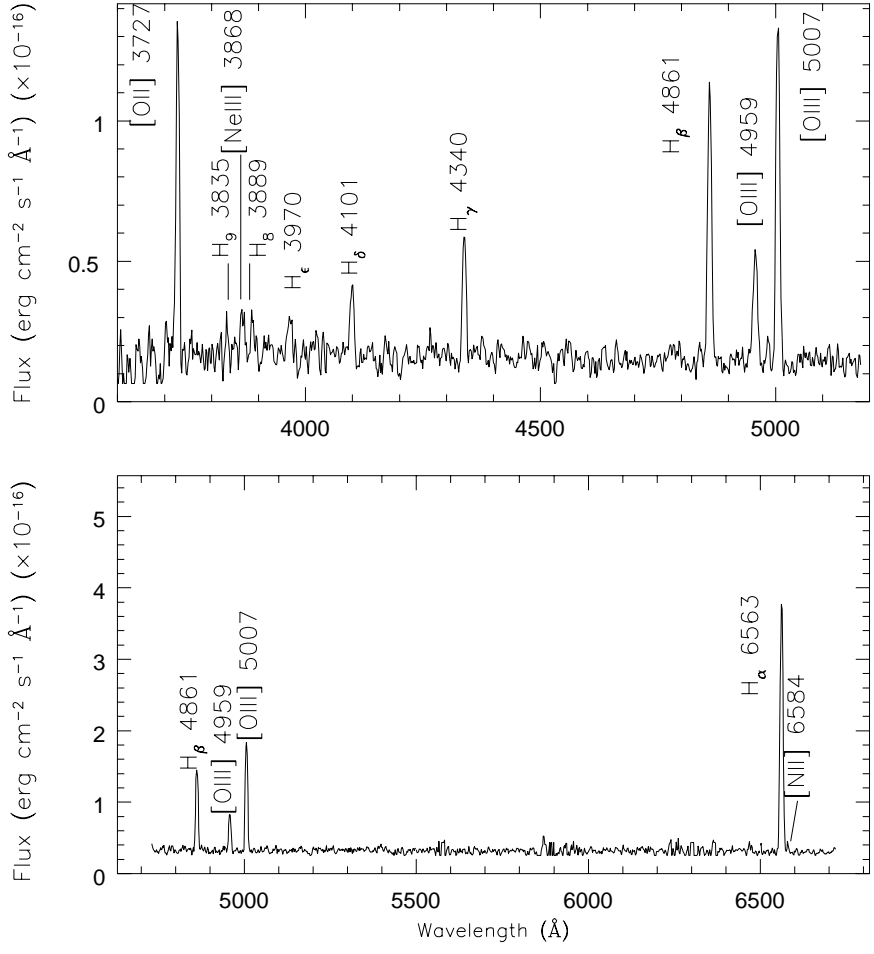

Fig. 1. The combined spectra of the brightest Hir region of SagDIG (object No.3 in Strobel et al. 1991). The upper panel shows the line identification for the averaged flux-calibrated spectrum obtained with grism \#7. The lower panel shows the same except for grism \#9.

temperature $T_{\mathrm{e}} \simeq 20000 \mathrm{~K}$. We set $R=I_{\lambda} / I_{\mathrm{H} \beta}, R_{0}=I_{\lambda, 0} / I_{\mathrm{H} \beta, 0}$ and $\varphi(\lambda)=[f(\lambda)-f(H \beta)]$ so that $C=\left[\log R_{0} / R\right] / \varphi(\lambda)$. The reddening constant was then computed using the Cardelli et al. (1989) extinction law, where $f(\lambda)=\langle A(\lambda) / A(V)\rangle$, and we assumed $R_{V}=3.1$. We obtained $C=0.335 \pm 0.061$ from the $\mathrm{H} \alpha / \mathrm{H} \beta$ ratio, and $C=0.67 \pm 0.82$ from the $\mathrm{H} \beta / \mathrm{H} \gamma$ ratio. As an alternate route, one can plot $\log \left(R_{0} / R\right)$ vs. $\varphi(\lambda)$ and then find $C$ with a linear regression on the data. A weighted least-square fit on the first three $\mathrm{H}$ lines yields $C=0.36 \pm 0.05$. Thus the value of the reddening constant is essentially fixed by the more accurate $\mathrm{H} \alpha / \mathrm{H} \beta$ ratio, and $C=0.335 \pm 0.061$ will be adopted in the rest of this paper. The reddening corrected flux ratios are listed in Table 1. When the same line is present in both the red and blue spectra, a weighted mean is computed before the correction is applied. In order to compute the final errors, the error contribution from the reddening correction was added in quadrature to the error on the flux.

\section{Results}

Chemical abundances were derived from these measurements using the IRAF nebular package (Shaw \& Dufour 1995). The abundances were calculated assuming a constant electron temperature and density throughout the nebula. For optical spectra covering the observed wavelength range, the electron temperature should be obtained through $T_{\mathrm{e}}$-sensitive line ratios, e.g. from the ratio of [OIII] lines $R_{4363}=[I(4959)+$ $I(5007)] / I(4363)$ (see Eq. (2.3) in Seaton 1975). However, the
Table 1. Line flux ratios relative to $\mathrm{H} \beta$.

\begin{tabular}{lllcc}
\hline \hline$\lambda(\AA)$ & Ident. & Grism \#7 & Grism \#9 & Redd. corrected \\
3727 & {$[$ OII $]$} & $1.02 \pm 0.20$ & - & $1.31 \pm 0.21$ \\
3835 & $\mathrm{H}_{9}$ & $0.08 \pm 0.15^{\mathrm{b}}$ & - & $0.10 \pm 0.16$ \\
3868 & {$[\mathrm{NeIII}]$} & $0.13 \pm 0.05$ & - & $0.16 \pm 0.08$ \\
3889 & $\mathrm{H}_{8}$ & $0.12 \pm 0.15^{\mathrm{b}}$ & - & $0.15 \pm 0.16$ \\
3970 & $\mathrm{H} \epsilon^{\mathrm{a}}$ & $0.17 \pm 0.15^{\mathrm{b}}$ & - & $0.21 \pm 0.16$ \\
4101 & $\mathrm{H} \delta$ & $0.19 \pm 0.07$ & - & $0.23 \pm 0.08$ \\
4340 & $\mathrm{H} \gamma$ & $0.36 \pm 0.11$ & - & $0.41 \pm 0.11$ \\
4861 & $\mathrm{H} \beta$ & $1.00 \pm 0.04$ & $1.00 \pm 0.03$ & $1.00 \pm 0.01$ \\
4959 & {$[\mathrm{OIII}]$} & $0.48 \pm 0.06$ & $0.46 \pm 0.04$ & $0.46 \pm 0.01$ \\
5007 & {$[\mathrm{OIII}]$} & $1.35 \pm 0.10$ & $1.34 \pm 0.02$ & $1.30 \pm 0.01$ \\
6563 & $\mathrm{H} \alpha$ & - & $3.58 \pm 0.03$ & $2.85 \pm 0.05$ \\
6584 & {$[\mathrm{NII}]$} & - & $0.15 \pm 0.06$ & $0.12 \pm 0.07$ \\
\hline
\end{tabular}

a Possible blend with 3968 [OIII].

${ }^{\mathrm{b}}$ Estimated maximum error (0.15) (see text).

$\lambda 4363$ line was not detected even in our co-added spectra, so we had to use the so-called empirical method, which gives the total $(\mathrm{O} / \mathrm{H})$ abundance in terms of the $R_{23}=[I(4959)+I(5007)+$ $I(3727)] / I(H \beta)$ parameter (Pagel et al. 1979; Edmunds \& Pagel 1984).

Oxygen abundances are plotted against $R_{23}$ in Fig. 2, using literature samples of HII regions spanning a wide range of abundances (Oey \& Shields 2000; Díaz \& Pérez-Montero 2000; Kennicutt et al. 2000; McGaugh 1991). In the same diagram, we show a set of cLoudy (Ferland et al. 1998) simulations, obtained assuming that the ionizing source is a single star with a Mihalas (1972) continuum, for 4 choices of the ionization parameter $(\log U=-1,-2,-3$ and -4$)$ and varying the metallicity from $12+\log (\mathrm{O} / \mathrm{H})=6.47$ to $12+\log (\mathrm{O} / \mathrm{H})=8.87$ (solar composition). The effects of a change in the temperature of the ionizing star were checked by running the simulations for both $T_{\text {eff }}=40000 \mathrm{~K}$ and $T_{\text {eff }}=50000 \mathrm{~K}$.

This figure, an updated version of Fig. 2 of Skillman (1989; hereafter S89) and Fig. 12 of McGaugh (1991), shows that, for abundances $12+\log (\mathrm{O} / \mathrm{H}) \leq 8, R_{23}$ depends both on the metallicity and the ionization parameter of the nebula. The S89 relation corresponds to the lower branch of the $\log U=-2$ models and to the most metal-poor objects in the data samples, irrespective of the effective temperature of the ionizing source.

Several authors have attempted to include both the effects of metallicity and ionization parameter in their empirical calibration methods. According to McGaugh (1991), the parameter $\log$ ([OIII]4959, 5007/[OII]3727) is useful to constrain $U$. More recently, Pilyugin (2000, 2001a, 2001b) has provided useful formulae to implement McGaugh corrections into the empirical method. Pilyugin (2001b; hereafter P01) transformed the $\log (\mathrm{O} / \mathrm{H})=f\left(R_{23}, \log U\right)$ relation into a $\log (\mathrm{O} / \mathrm{H})=$ $h\left(R_{23}, R_{3}\right)$ relation, where $R_{3}=\log ([\mathrm{OIII}] 4959,5007 / \mathrm{H} \beta)$. In this case he gives the equation of the surface, which is:

$12+\log (\mathrm{O} / \mathrm{H})=6.35+3.19 \times \log \left(R_{23}\right)-1.74 \times \log \left(R_{3}\right)$.

These revised methods may have problems at low values of the abundance and the ionization parameter. For example, the 


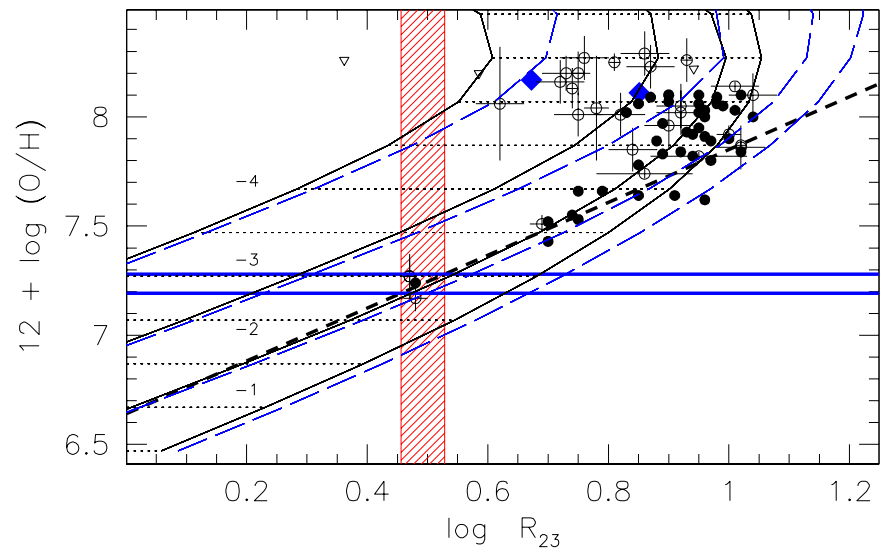

Fig. 2. $(\mathrm{O} / \mathrm{H})$ determination for SagDIG using the empirical method based on $R_{23}$. The $1 \sigma$ range of variation of our value of $\log \left(R_{23}\right)$ is shown by the vertical band. The empirical relation of Skillman (1989) is represented by the inclined heavy short-dashed line. The horizontal solid lines show the range of uncertainty of $\log (\mathrm{O} / \mathrm{H})$. For comparison, values of $\log (\mathrm{O} / \mathrm{H})$ are plotted against the value of $\log R_{23}$ for the sample of HII regions from Díaz \& Pérez-Montero (2000) (open circles), Oey \& Shields (2000) (filled diamonds), Kennicutt et al. (2000) (open triangles), and McGaugh (1991) (filled circles). Only objects more metal poor than $12+\log (\mathrm{O} / \mathrm{H})=8.3$ are plotted. A number of CLoudy simulations are also shown. The thin solid curves represent the theoretical relations obtained by varying the ionization parameter from $\log U=-1$ to $\log U=-4$ (see labels), and by varying $[\mathrm{m} / \mathrm{H}]$ from -0.4 to -2.4 (top to bottom, dotted lines). An input ionizing spectrum from a $T_{\text {eff }}=40000 \mathrm{~K}$ star was assumed. The effect of changing the input spectrum is shown by the long-dashed curves, which were calculated for $T_{\text {eff }}=50000 \mathrm{~K}$.

dwarf galaxies ESO 380-27 and GR 8 have similar $\log \left(R_{23}\right)$, but $\log \left(R_{3}\right)=0.67$ for the former, and $\log \left(R_{3}\right)=0.43$ for the latter. The low excitation parameter then implies that GR 8 should have a lower $\log U$, implying a higher oxygen abundance. Indeed, we find $12+\log (\mathrm{O} / \mathrm{H})=7.58$ for the first, and $12+\log (\mathrm{O} / \mathrm{H})=7.83$ for the second galaxy. The corrected empirical method would then predict that GR 8 should have an oxygen abundance 0.25 dex higher than ESO 380-27, but in reality the measured abundance from the temperature determinations is 0.10 dex lower in GR 8 than in ESO 380-27 (see Kunth \& Sargent 1983; Skillman et al. 1988). Given all these uncertainties, we used both the original implementation of the empirical method (as in STM89) and more recent methods to provide a range of $[\mathrm{O} / \mathrm{H}]$ for SagDIG. High $S / N$ spectra taken at $10-\mathrm{m}$ class telescopes will be needed to place better constraints on the SagDIG abundance, by allowing the measurement of the $\lambda 4363$ [OIII] line.

Therefore, we firstly used the relative $\mathrm{O}$ fluxes listed in Table 1 to obtain $R_{23}=3.12 \pm 0.26$ (i.e. $0.46 \leq \log R_{23} \leq 0.53$ ). The calibration of $\mathrm{S} 89$ gives $7.19 \leq 12+\log (\mathrm{O} / \mathrm{H}) \leq 7.28$, where the range in variation has been obtained by propagating the error on $R_{23}$. An application of the empirical calibration therefore indicates a very low oxygen abundance for the Sagittarius dwarf irregular galaxy. This value can be directly compared to the abundance obtained by STM 89 using the same calibration. The new abundance estimate is lower by $\sim 0.2 \mathrm{dex}$, and the difference is real and traced to the new flux measure-

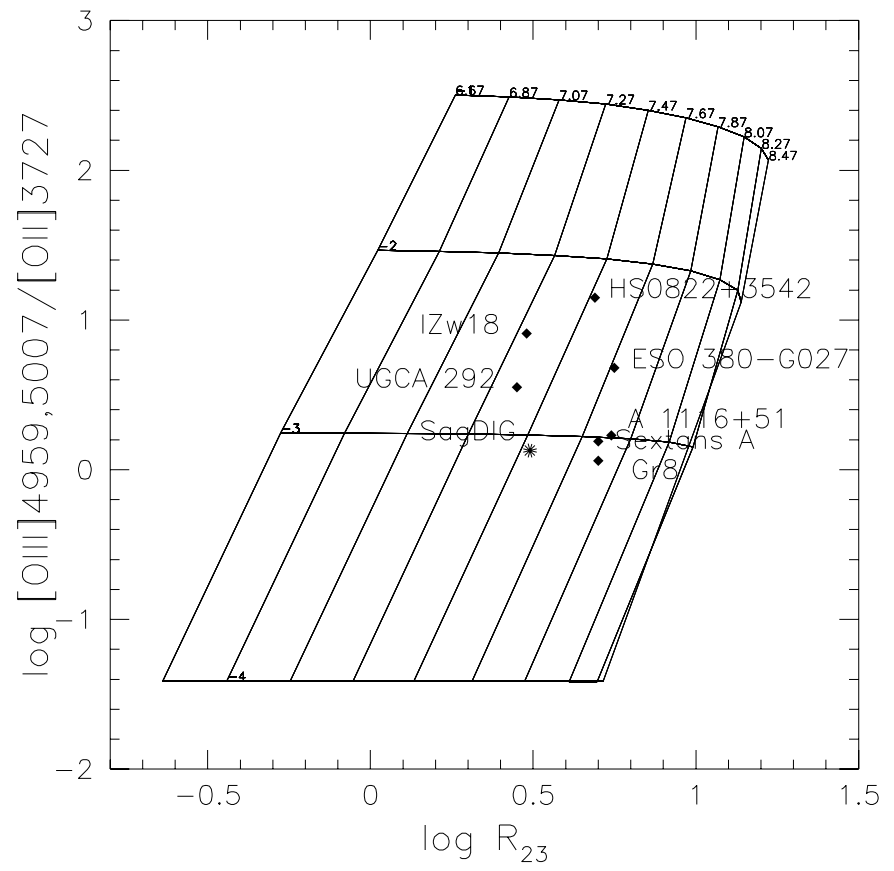

Fig. 3. A comparison of the observed values of $R_{23}$ and the [OIII]/[OII] ratio for the most metal-poor star-forming dwarfs with a model grid obtained from cLoudy simulations, for $T_{\text {eff }}=50000 \mathrm{~K}$. The data are from Skillman (1989), McGaugh (1991), Van Zee (1999), and Kniazev et al. (2000).

ments. Since our measurements from the red and blue EFosc2 spectra are perfectly consistent, whereas the measurements of STM89 presented a $\sim 40 \%$ internal scatter, we are confident in our revised fluxes (indeed, using only the STM89 red spectrum for the $\lambda \lambda 4959,5007$ fluxes, we would obtain a value $12+\log (\mathrm{O} / \mathrm{H})=7.22$, in perfect agreement with ours).

Secondly, the revisions of the empirical method were taken into account by plotting in Fig. 3 the [OIII]/[OII] ratio against the $R_{23}$ for a subsample of very metal-poor dwarfs (see Fig. 10 in McGaugh 1991). The data are overplotted onto a model grid derived from the same cLoudy models as in Fig. 2. In the case of SagDIG, we have $\log ([$ OIII $] 4959,5007 /[$ OII] 3727$) \simeq 0.13$, which implies $\log U \approx-3$. The position of SagDIG with respect to the lines of constant $\mathrm{O}$ abundance indicates a metallicity intermediate between those of IZw 18 and GR 8, and close to that of HS0822+3542, for which Kniazev et al. (2000) give $12+\log (\mathrm{O} / \mathrm{H})=7.35$. Thus, taking into account in a differential way the information provided by the ionization indicator [OIII]/[OII], we find $12+\log (\mathrm{O} / \mathrm{H}) \simeq 7.35$.

Finally, we evaluated the $\mathrm{O}$ abundance by using cLoudy to optimize the fit to the observed line ratios. The best-fit is obtained for a value $12+\log (\mathrm{O} / \mathrm{H})=7.41$, i.e. $1 / 29$ solar, with an electron temperature $T_{\mathrm{e}} \sim 16000 \mathrm{~K}$ and $\log U \simeq-2.8$. Not surprisingly, this result is consistent with that inferred from Fig. 3.

To proceed further to estimate the $\mathrm{N}$ and $\mathrm{Ne}$ abundances in SagDIG, we need making some considerations (and assumptions) on the electron temperature and density in the nebula. A loose upper limit on the electron temperature is set by non-detection of the $\lambda 4363$ [OIII] line. Experiments simulating faint emission lines yield a $2 \sigma$ upper limit of 0.09 
on the flux of the $\lambda 4363$ line relative to $\mathrm{H} \beta$, which implies $T_{\mathrm{e}}<34000 \mathrm{~K}$ according to the Seaton's (1975) relation. A temperature $T_{\mathrm{e}}=20000 \mathrm{~K}$ is needed in the nebular calculations to obtain an $(\mathrm{O} / \mathrm{H})$ value compatible with the results from the empirical method (assuming $N_{\mathrm{e}}=1000 \mathrm{~cm}^{-3}$ ). In fact, using these values we obtain $N\left(\mathrm{O}^{+}\right) / \mathrm{N}\left(\mathrm{H}^{+}\right)=(1.09 \pm$ $0.17) \times 10^{-5}$ and $N\left(\mathrm{O}^{++}\right) / \mathrm{N}\left(\mathrm{H}^{+}\right)=(0.73 \pm 0.01) \times 10^{-5}$, yielding $\left(\mathrm{O}^{+}+\mathrm{O}^{++}\right) / \mathrm{H}^{+}=(1.82 \pm 0.18) \times 10^{-5}$, (i.e. $12+\log \left[\left(\mathrm{O}^{+}+\right.\right.$ $\left.\left.\left.\mathrm{O}^{++}\right) / \mathrm{H}^{+}\right]=7.26\right)$. This choice of physical parameters predicts $R_{4363} \simeq 37$, implying that the $\lambda 4363$ line flux ratio should be $\simeq 0.05$, definitely lower than the minimum normalized flux that we have been able to measure.

Yet, the low surface brightness of the nebula seems to suggest a lower electron temperature and ionization parameter, in accord with the above cLoudy model calculations. In view of these uncertainties, we adopt $T_{\mathrm{e}}=18000 \pm 2000$ for electron temperature, implying $12+\log (\mathrm{O} / \mathrm{H})=7.37_{-0.11}^{+0.13}$ for the $\mathrm{O}$ abundance in SagDIG, in accord with the different empirical approaches discussed above. For the nitrogen and neon ionic abundances in UKS 1927-177, $\left(\mathrm{N}^{+} / \mathrm{H}^{+}\right)=$ $(0.062 \pm 0.039) \times 10^{-5}$ and $\left(\mathrm{Ne}^{++} / \mathrm{H}^{+}\right)=(0.284 \pm 0.134) \times 10^{-5}$ are derived. The total abundances can be computed following Peimbert \& Costero (1969) (see also van Zee 1998), obtaining $12+\log (\mathrm{N} / \mathrm{H})=6.00_{-0.98}^{+0.28}$ and $12+\log (\mathrm{Ne} / \mathrm{H})=6.86_{-0.38}^{+0.20}$. This also implies $\log (\mathrm{N} / \mathrm{O})=-1.36_{-1.76}^{+0.30}$ and $\log (\mathrm{Ne} / \mathrm{O})=$ $-0.50_{-0.51}^{+0.22}$. The quoted uncertainties for the $\mathrm{N}$ and $\mathrm{Ne}$ abundances reflect the measurements errors and are larger than those related to the temperature range. Notwithstanding the above uncertainties, this represents the first measurement of $\mathrm{N}$ and $\mathrm{Ne}$ abundances in UKS 1927-177.

\section{Discussion}

Our results confirm that SagDIG is indeed one of the most metal poor galaxies, having abundances just $50 \%$ higher than I Zw 18. In the Local Group, only Leo A has a comparably low metallicity. The nitrogen abundance of SagDIG is consistent with that of IZw 18 (Izotov \& Thuan 1998). For Leo A, van Zee et al. (1999) report a nitrogen abundance $\log \mathrm{N} / \mathrm{O}=-1.5 \pm 0.1$, similar to other low-metallicity galaxies.

Answering the question about whether SagDIG and Leo A are young galaxies rests on the results of resolved photometry. For SagDIG, ground based observations indicate the presence of an intermediate-age stellar population in addition to the young stars (Karachentsev et al. 1999; Momany et al. 2001), but the presence of a really old component is still unproven. As is the case of Leo I, where wide-field observations have revealed an old HB (Held et al. 2000, 2001), deep HsT observations of SagDIG may eventually answer the original question of how old this metal-poor galaxy is.

In Leo A, Tolstoy et al. (1998) could not rule out the presence of such a population in their HST photometry, although it must be a minor component. Recently, Dolphin et al. (2002) have detected a few candidate RR Lyrae variable stars, indicating the presence of an old (globular cluster like) stellar population accounting for at least $0.1 \%$ of the galaxy's luminosity.

Finally, it is important to check that the low metal content of SagDIG is the result of normal chemical evolution, and not, for example, of gas stripping by a massive neighbor. Indeed, its position at the margins of the Local Group seems to ensure self-regulated evolution, an idea that we reinforce by the following simple considerations of chemical evolution.

In the closed-box model, the expected ISM oxygen abundance is given by $(\mathrm{O} / \mathrm{H})=(\mathrm{O} / \mathrm{H})_{\mathrm{y}} \ln (1 / \mu)$ (Searle \& Sargent 1972). A "standard" value for the yield of oxygen, $(\mathrm{O} / \mathrm{H})_{\mathrm{y}}$, is $2 / 3$ of the solar abundance, i.e. $(\mathrm{O} / \mathrm{H})_{\mathrm{y}}=4.9 \times 10^{-4}$ (e.g. Pagel $1997)$; and $\mu=G /(G+S)$ is the ratio of the gas mass $G$ over total mass ( $S$ is the mass in stars).

The gas mass given by Young \& Lo (1997) is $1.3 \times 10^{7} M_{\odot}$, and the mass in stars and remnants, computed by integrating the SFR given by Karachentsev et al. (1999) in three age intervals, is $2 \times 10^{6} M_{\odot}$. We thus obtain $\mu=0.86$, a value that in the closed-box model implies $12+\log (\mathrm{O} / \mathrm{H})=7.87$, higher than the measured one. We would need $\mu=0.97$ to reproduce our observation. The observed lower $\mathrm{O}$ abundance could be explained if part of the enriched gas is lost into the intergalactic medium. With some algebra, we find that the mass lost should be $\Delta G=0.11 \times G=1.5 \times 10^{6} M_{\odot}$. According to Karachentsev et al. (1999), the SFR during the last $200 \mathrm{Myr}$ was $\sim 10$ times higher than in the past, with a further enhancement during the last $50 \mathrm{Myr}$. If we assume that the mass loss occurred during the last 200 million years, then we obtain a mass outflow of $\dot{M}=0.01 M_{\odot} \mathrm{yr}^{-1}$, while $\dot{M}=0.03 M_{\odot} \mathrm{yr}^{-1}$ if the mass outflow occurred during the last 50 million years. These are not unreasonable values, since for example outflows of the order of $0.2 \div 2 M_{\odot} \mathrm{yr}^{-1}$ are observed in the case of NGC 1705 (Meurer et al. 1998), another isolated dwarf galaxy.

Using the higher value of the gas fraction, we find an average metallicity for the stellar component which is in agreement with the values derived from broadband photometry. Using Eq. (8.7) of Pagel (1997), an average oxygen abundance of 0.01 solar is obtained for the stars. For solar-scaled metal abundances, this would mean a metallicity $[\mathrm{Fe} / \mathrm{H}]=-2$, which agrees with the stellar metallicity measured by Momany et al. (2001), $[\mathrm{Fe} / \mathrm{H}] \simeq-2.1$.

Our conclusion that a very metal-poor ISM is present in SagDIG goes in the direction of the accumulating evidence that very low oxygen abundances can be maintained throughout the life of a dwarf galaxy, even if it is not experiencing its first star formation episode, and without invoking special environmental conditions. It is fairly common that galaxies once believed to contain only a young population actually reveal an old halo when modern data are obtained (see Kunth \& Östlin 2000 for a review; also Saviane et al. 2001).

Acknowledgements. We thank Michael Sterzik for his precious help with the observations, and Stefano Ciroi for sharing with us his expertise in cLoudy. We also thank the referee, Dr. C. Leitherer, for helpful comments.

\section{References}

Allende Prieto, C., Lambert, D. L., \& Asplund, M. 2001, ApJ, 556, L63

Aloisi, A., Tosi, M., \& Greggio, L. 1999, AJ, 118, 302

Anders, E., \& Grevesse, N. 1989, Geochim. Cosmochim. Acta, 53, 197 
Cardelli, J. A., Clayton, G. C., \& Mathis, J. S. 1989, ApJ, 345, 245

Cesarsky, D. A., Lequeux, J., Laustsen, S., Schuster, H.-E., \& West, R. M. 1977, A\&A, 61, L31

Cook, K. H. 1987, Ph.D. Thesis, University of Arizona

Díaz, A. I., \& Pérez-Montero, E. 2000, MNRAS, 312, 130

Dolphin, A. E., Saha, A., Claver, et al. 2002, AJ, in press [astro-ph/0202381]

Edmunds, M. G., \& Pagel, B. E. J. 1984, MNRAS, 211, 507

Ferland, G. J., Korista, K. T., Verner, D. A., et al. 1998, PASP, 110, 761

Hamuy, M., Walker, A. R., Suntzeff, N. B., et al. 1992, PASP, 104, 533

Hamuy, M., Suntzeff, N. B., Heathcote, S. R., et al. 1994, PASP, 106, 566

Held, E. V., Saviane, I., Momany, Y., \& Carraro, G. 2000, ApJ, 530, L85

Held, E. V., Clementini, G., Rizzi, L., et al. 2001, ApJ, 562, L39

Hidalgo-Gamez, A. M., \& Olofsson, K. 1998, A\&A, 334, 45

Holweger, H. 2001, in Proceedings of the Joint SOHO/ACE workshop Solar and Galactic Composition, ed. R. F. Wimmer-Schweingruber, AIP Conf. Proc., 598, 23

Hummer, D. G., \& Storey, P. J. 1987, MNRAS, 224, 801

Hunter, D. A., \& Hoffman, L. 1999, AJ, 117, 2789

Izotov, Y. I., \& Thuan, T. X. 1998, ApJ, 497, 227

Izotov, Y. I., Chaffee, F. H., Foltz, C. B., et al. 2001, ApJ, 560, 222

Karachentsev, I., Aparicio, A., \& Makarova, L. 1999, A\&A, 352, 363

Kennicutt, R. C., Bresolin, F., French, H., \& Martin, P. 2000, ApJ, 537, 589

Kniazev, A. Y., Pustilnik, S. A., Masegosa, J., et al. 2000, A\&A, 357, 101

Kunth, D., \& Östlin, G. 2000, A\&A Rev., 10, 1

Kunth, D., \& Sargent, W. L. W. 1983, ApJ, 273, 81

Lee, M. G., \& Kim, S. C. 2000, AJ, 119, 777

Mc Gaugh, S. 1991, ApJ, 380, 140

Meurer, G. R., Staveley-Smith, L., \& Killeen, N. E. B. 1998, MNRAS, 300, 705

Mihalas, D. 1972, Non-LTE Model Atmospheres for B \& O Stars, NCAR-TN/STR-76
Momany, Y., Held, E. V., Saviane, I., \& Rizzi, L. 2001, A\&A, 384, 393

Oey, M. S., \& Shields, J. C. 2000, ApJ, 539, 687

Oke, J. B. 1990, AJ, 99, 1621

Osterbrock, D. E. 1989, Astrophysics of Gaseous Nebulae and Active Galactic Nuclei (Mill Valley: Univ. Sci.)

Pagel, B. E. J. 1997, Nucleosynthesis and Chemical Evolution of Galaxies (Cambridge: Cambridge University Press)

Pagel, B. E. J., Edmunds, M. G., Blackwell, D. E., Chun, M. S., \& Smith, G. 1979, MNRAS, 189, 95

Peimbert, M., \& Costero, R. 1969, Bol. Obs. Tonantzintla y Tacubaya, 5,3

Pilyugin, L. S. 2000, A\&A, 362, 325

Pilyugin, L. S. 2001a, A\&A, 369, 594

Pilyugin, L. S. 2001b, A\&A, 374, 412

Saviane, I., Held, E. V., Momany Y., \& Rizzi, L. 2001, in Proceedings of the 44th National Congress of the Italian Astron. Society, ed. L. A. Antonelli, G. Bono, G. Giobbi, \& N. Menci, Mem.S.A.It. 72 (4), 773

Searle, L., \& Sargent, W. L. W. 1972, ApJ, 173, 25

Seaton, M. J. 1975, MNRAS, 170, 475

Shaw, R. A., \& Dufour, R. J. 1995, PASP, 107, 896

Skillman, E. D. 1989, ApJ, 347, 883 (S89)

Skillman, E. D., Kennicutt, R. C., \& Hodge, P. W. 1989, ApJ, 347, 875

Skillman, E. D., Terlevich, R., \& Melnick, J. 1989, MNRAS, 240, 563 (STM89)

Skillman, E. D., Melnick, J., Terlevich, R., \& Moles, M. 1988, MNRAS, 230, 563

Strobel, N. V., Hodge, P., \& Kennicutt, R. C. 1991, ApJ, 383, 148 (SHK91)

Tolstoy, E., Gallagher, J. S., Cole, A. A., et al. 1998, AJ, 116, 1244

van Zee, L. 2000, ApJ, 543, L31

van Zee, L., Salzer, J. J., Haynes, M. P., O’Donoghue, A. A., \& Balonek, T. J. 1998, AJ, 116, 2805

van Zee, L., Skillman, E. D., \& Haynes, M. P. 1999, AAS, 194, \#05.04

Young, L. M., \& Lo, K. Y. 1997, ApJ, 490, 710 